\title{
Karhunen-Loeve Representation of Periodic Second-Order Autoregressive Processes
}

\author{
Didier Lucor, Chau-Hsing Su, and George Em Karniadakis \\ Division of Applied Mathematics, Brown University, Providence, RI 02912, USA.
}

\begin{abstract}
In dynamic data driven applications modeling accurately the uncertainty of various inputs is a key step of the process. In this paper, we first review the basics of the Karhunen-Loève decomposition as a means for representing stochastic inputs. Then, we derive explicit expressions of one-dimensional covariance kernels associated with periodic spatial second-order autoregressive processes. We also construct numerically those kernels by employing the Karhunen-Loève expansion and making use of Fourier representation in order to solve efficiently the associated eigenvalue problem. Convergence and accuracy of the numerical procedure are checked by comparing the covariance kernels obtained from the Karhunen-Loève expansions against theoretical solutions.
\end{abstract}

\section{Introduction}

In many application of dynamic data driven systems there is a significant degree of uncertainty associated with the various inputs due to inaccuracy of measurements, gappy data, or incomplete knowledge. We can incorporate this uncertainty directly into the models by formulating stochastic algorithms, but a significant first step is to represent accurately and efficiently all stochastic inputs. The Karhunen-Loève (KL) expansion is a very powerful tool for representing stationary and non-stationary random processes with explicitly known covariance functions [1. In a few cases, the eigen-solutions of the covariance function can be obtained analytically but in most cases they have to be obtained numerically [2. The KL representation is optimal as both the deterministic basis functions and the corresponding random coefficients are orthogonal. If the random process is assumed stationary and the ratio between the length of the domain and the correlation length is infinite or periodic boundary conditions are imposed, the KL representation becomes a spectral expansion with uncorrelated random coefficients 3 .

One of the simplest and most used random processes is the first-order Markov process, which relates to the Brownian motion of small particles and the diffusion phenomenon. It is a unilateral type of scheme extended only in one direction. The covariance kernel associated with that one-dimensional first-order autoregressive process takes an exponential form [2]. However, realistic models of random series in space require autoregressive schemes with dependence in all directions. In some cases, it has been shown that schemes of bilateral type in one dimension can be effectively reduced to a unilateral one [4]. 
In the current work, we derive explicit expressions of one-dimensional covariance kernels associated with periodic second-order autoregressive processes. We then represent numerically those kernels using a KL representation where we make use of the Fourier series representation in order to compute efficiently the associated eigenvalue problem. Convergence and accuracy of the numerical method are checked against theoretical solutions for the kernels.

\section{The Karhunen-Loève Expansion}

The KL expansion is based on the spectral expansion of the covariance function $c\left(t_{1}, t_{2}\right)$ of the process $v(t, \theta)$, where $t_{1}$ and $t_{2}$ are two temporal coordinates and time is from $a$ to $b$. The KL expansion then takes the following form:

$$
v(t, \theta)=\bar{v}(t)+\sigma_{v} \sum_{i=1}^{\infty} \sqrt{\lambda_{i}} \psi_{i}(t) \xi_{i}(\theta),
$$

where $\bar{v}(t)$ denotes the mean of the random process, $\sigma_{v}$ denotes the standard deviation of the process, and $\xi_{i}(\theta)$ is a set of independent random variables with a given random distribution; they form an orthonormal random vector (throughout this paper, we will use the symbol $\xi$ to denote a random variable with zero mean and unit variance.) Obviously, truncated expansions will be used in practice. Also, $\psi_{i}(t)$ and $\lambda_{i}$ are the eigenfunctions and eigenvalues of the covariance function, respectively, i.e.,

$$
\int_{a}^{b} c\left(t_{1}, t_{2}\right) \psi_{i}\left(t_{2}\right) d t_{2}=\lambda_{i} \psi_{i}\left(t_{1}\right) .
$$

The KL expansion minimizes the mean-square error resulting from a finite-term representation of the process [2]. Its use, however, is limited as the covariance function of the solution process is not known a priori. Nevertheless, the KL expansion still provides a powerful means for representing input random processes when the covariance structure is known. Some numerical experiments regarding the accuracy of truncated KL representation for different covariance kernels have been reported in 56 . The rate of convergence of the KL expansion is closely related to the smoothness of the covariance kernel and to the ratio between the length of process $T$ and the correlation length $A$. For instance, for the particular case of a first-order Markov process, given by $c\left(t_{1}, t_{2}\right)=\sigma_{v}^{2} e^{-\left|t_{2}-t_{1}\right| / A}$, an upper bound for the relative error in variance $\epsilon$ of the process represented by its $\mathrm{KL}$ expansion is such that:

$$
\epsilon \leq \frac{4}{\pi^{2}} \frac{1}{n} \frac{T}{A} \approx 0.4053 \frac{1}{n} \frac{T}{A} .
$$

We see that it depends on the ratio $\frac{T}{A}$, where $T$ is the length of the time domain, and it is inversely proportional to the number of retained terms. The dependence in $\left(\frac{1}{n}\right)$ is related to the rate of convergence of the Fourier decomposition for this particular kernel, which has a discontinuity in its first derivative at the origin [6]. 


\section{Fourier Series Representation of the Integral Equation}

If the random process is stationary with respect to $t$, then the covariance satisfies:

$$
c\left(t, t_{1}\right)=\left\langle v(t) v\left(t_{1}\right)\right\rangle=c\left(t-t_{1}\right) .
$$

The corresponding integral equation (2) for the eigenfunctions and eigenvalues used in the KL representation can be cast into a spectral form by taking a Fourier series representation of the kernel function $c\left(t-t_{1}\right)$ and the eigenfunctions $\psi(t)$. The Fourier series representation provides a natural way of solving the problem if it is periodic, i.e. both the kernel function $c$ and the eigenfunctions $\psi$ are periodic functions with period $T$. In this case, the integral equation can be solved exactly.

With both $\psi(t)$ and $c(t)$ being periodic functions of period $\mathrm{T}$, we represent them in terms of their Fourier series as follows:

$$
\begin{aligned}
& \psi(t)=\frac{\psi_{c o}}{2}+\sum_{n=1}^{\infty}\left[\psi_{c n} \cos \frac{2 n \pi}{T}(t-a)+\psi_{s n} \sin \frac{2 n \pi}{T}(t-a)\right], \\
& c(t)=\frac{c_{c o}}{2}+\sum_{n=1}^{\infty}\left[c_{c n} \cos \frac{2 n \pi}{T} t+c_{s n} \sin \frac{2 n \pi}{T} t\right]
\end{aligned}
$$

with

$$
\begin{aligned}
\psi_{c n} & =\frac{2}{T} \int_{a}^{b} d t \psi(t) \cos \frac{2 n \pi}{T}(t-a), \quad \psi_{s n}=\frac{1}{T} \int_{a}^{b} d t \psi(t) \sin \frac{2 n \pi}{T}(t-a) \\
c_{c n} & =\frac{2}{T} \int_{-T / 2}^{T / 2} d t c(t) \cos \frac{2 n \pi}{T} t, \quad c_{s n}=\frac{2}{T} \int_{-T / 2}^{T / 2} d t c(t) \sin \frac{2 n \pi}{T} t
\end{aligned}
$$

In most applications $c(t)$ is an even function and thus $c_{s n}=0$. Substituting equations (5.6) into equation (2), we obtain:

$$
\left(\lambda-\frac{c_{c o}}{2} T\right) \frac{\psi_{c o}}{2}+\sum_{n=1}^{\infty}\left(\lambda-\frac{c_{c n}}{2} T\right)\left[\psi_{c n} \cos \frac{2 n \pi}{T}(t-a)+\psi_{s n} \sin \frac{2 n \pi}{2}(t-a)\right]=0 .
$$

We derive the set of eigenvalues and eigenfunctions as:

1. $\lambda=\lambda_{0}=\frac{T}{2} c_{c o}, \psi_{c 0} \neq 0, \psi_{c n}=\psi_{s n}=0$ for $n \neq 0$ and $\psi^{(0)}=\frac{1}{\sqrt{T}}$.

2. $\lambda=\lambda_{n}=\frac{T}{2} c_{c n}, \psi_{c n} \neq 0$, and $\psi_{s n} \neq 0, \psi_{c m}=\psi_{s m}=0$ for $m \neq n$, and

$$
\psi_{1}^{(n)}\left(t_{1}\right)=\sqrt{\frac{2}{T}} \cos \frac{2 n \pi}{T}\left(t_{1}-a\right), \quad \psi_{2}^{(n)}\left(t_{1}\right)=\sqrt{\frac{2}{T}} \sin \frac{2 n \pi}{T}\left(t_{1}-a\right) .
$$

The KL representation of the periodic random process $v(t, \xi)$ is:

$$
v(t, \xi)=\sqrt{\frac{\lambda_{o}}{T}} \xi_{c o}+\sqrt{\frac{2}{T}} \sum_{n=1}^{\infty} \sqrt{\lambda_{n}}\left[\xi_{c n} \cos \frac{2 n \pi}{T}(t-a)+\xi_{s n} \sin \frac{2 n \pi}{T}(t-a)\right],
$$


where

$$
\lambda_{n}=\frac{T}{2} c_{c n}=\int_{-T / 2}^{T / 2} c(t) \cos \frac{2 n \pi}{T} t d t=2 \int_{0}^{T / 2} c(t) \cos \frac{2 n \pi}{T} t d t .
$$

Given a covariance function $c(t)$, one can solve these integrals using appropriate numerical quadrature methods. The corresponding covariance function which can be obtained directly from equation (11):

$$
\left\langle v(t, \xi) v\left(t_{1}, \xi\right)\right\rangle=\frac{c_{c o}}{2}+\sum_{n=1}^{\infty} c_{c n} \cos \frac{2 n \pi}{T}\left(t-t_{1}\right)=c\left(t-t_{1}\right),
$$

is just the Fourier series representation of the covariance function $c$.

It is worthwhile to note that this approach can also be used as an approximation for non-periodic cases if the domain $T$ of the problem is much larger than the correlation length $A$. The range of the functions $\psi(t)$ and $\psi\left(t_{1}\right)$ is $(a, b)$ while that of $c\left(t-t_{1}\right)$ is $(-T, T)$ where $T=b-a$. In order to represent the extended functions as Fourier series, i.e., we need to take the period to be $2 T$. However, if $c\left(t-t_{1}\right)$ has a compact support, or if it is different from zero only for $\left|t-t_{1}\right| \leq A$ for some finite $A$, then in cases where $A \ll T$, the relative error in using the period being $T$ rather than $2 T$ is of the order of $A / T$. In the literature, this approximation is sometime refered to as spectral approximation 78 .

Notations and more detailed derivations for Section 3 can be found in the work of Lucor 9].

\section{Second-Order Autoregressive Processes}

For space series, a bilateral autoregression takes the form:

$$
\begin{aligned}
v_{i} & =\gamma v_{i-1}+\delta v_{i+1}+\epsilon_{i} \quad \text { or } \\
v_{i} & =\frac{b}{2}\left(v_{i-1}+v_{i+1}\right)+a f \xi_{i},
\end{aligned}
$$

where it is intuitively clear that $\gamma$ and $\delta$ cannot be too large. Let us denote by $L$ the spatial length of the periodic domain and take $L=1$ without loss of generality. We discretize the domain with $2 m+1$ equidistant points, so that we have: $m=L / 2 \Delta x$. The expression for $v_{i}$, due to the homogeneity of the system based on the assumed periodicity, reduces to:

$$
v_{i}=\sum_{j=0}^{2 m-1} \alpha_{j} \xi_{i+j}
$$

The coefficients are such that:

$$
\alpha_{j}= \begin{cases}\frac{a f}{1-\frac{b^{2}}{D_{m}}}=1 & \text { for } j=0 \\ \alpha_{j-1} / D_{m-i+1} & \text { for } 0<j \leq i \text { with } D_{1}=1 ; D_{k}=2-b^{2} / D_{k-1} ; 1<k \leq m \\ 0 & \text { for } j>i\end{cases}
$$


Because we take $\alpha_{0}=1$, the variance of the process is conserved and is constant for each point in the domain, so we have: $a f=1-b^{2} / D_{m}$. The covariance $\left\langle v_{i} v_{j}\right\rangle$ between two points of the grid is obtained from the dynamical system:

$$
\left\langle v_{i} v_{j}\right\rangle=\sum_{j=0}^{2 m-1} \alpha_{j} \alpha_{j+i-k}
$$

Let us define the mode number $q$ that represents the number of waves that one could fit within the domain $L$; the corresponding wave number is $k=2 \pi q / L$. There exists an analogy between a second-order regressive dynamical system of type (14) and the discrete version of a non-homogeneous second-order ordinary equation [10]:

$$
\frac{v_{i+1}-2 v_{i}+v_{i-1}}{\Delta x^{2}} \pm k^{2} v_{i}=F\left(x_{i}\right) .
$$

Indeed, the system of equations (14) can be transformed into equation (16) by setting $b=1 /\left(1 \pm \frac{1}{2}(k \Delta x)^{2}\right)$ and $a f \xi_{i}=-F\left(x_{i}\right) \Delta x^{2} /\left(2+(k \Delta x)^{2}\right)$. This gives in the continuous limit of $\Delta x$ going to zero:

$$
b=e^{ \pm \frac{1}{2}(k \Delta x)^{2}}=e^{ \pm \frac{1}{2}\left(\frac{2 \pi q}{L} \Delta x\right)^{2}}
$$

The wave number $k$ has the dimension of the inverse of a correlation length $A$, $A=1 / k$. The coefficient $b$ tends to unity as $\Delta x$ (grid size) tends to zero. The plus or minus signs in equation (17) indicate that $b$ can approach unity from above or below. This distinction gives totally different results for the covariance kernel form.

If $b=e^{-\frac{1}{2}(k \Delta x)^{2}}<1$, which corresponds to equation with a negative sign, and under the assumption that the domain is periodic, the covariance of the solution is:

$$
\begin{gathered}
\langle v(x), v(y)\rangle=\frac{1}{4 k^{2} \sinh ^{2} \frac{k L}{2}} \int_{0}^{L} \int_{0}^{L}\left\langle F\left(x_{1}\right) F\left(x_{2}\right)\right\rangle \cosh k\left(\left|x-x_{1}\right|-\frac{L}{2}\right) d x_{1} \\
\cosh k\left(\left|y-x_{2}\right|-\frac{L}{2}\right) d x_{2} .
\end{gathered}
$$

If the variance of the forcing function is conserved for each point of the domain, i.e. $\langle F(x) F(y)\rangle=\sigma_{F}^{2} \delta(x-y)$, it becomes:

$$
\langle v(x), v(y)\rangle=\frac{\sigma_{F}^{2}}{4 k^{2} \sinh ^{2} \frac{k L}{2}} \int_{0}^{L} \cosh k\left(\left|x-x_{1}\right|-\frac{L}{2}\right) \cosh k\left(\left|y-x_{1}\right|-\frac{L}{2}\right) d x_{1} .
$$

The normalized covariance kernel becomes in this case:

$$
\begin{aligned}
c(x, y)= & \frac{1}{2 \alpha+\sinh (2 \alpha)}[2 \alpha+\sinh (2 \alpha)+\alpha|x-y|(\cosh (2 \alpha)-1)] \cosh (\alpha|x-y|) \\
& +[1-\cosh (2 \alpha)-\alpha|x-y| \sinh (2 \alpha)] \sinh (\alpha|x-y|) \quad ; \quad \alpha=q \pi
\end{aligned}
$$



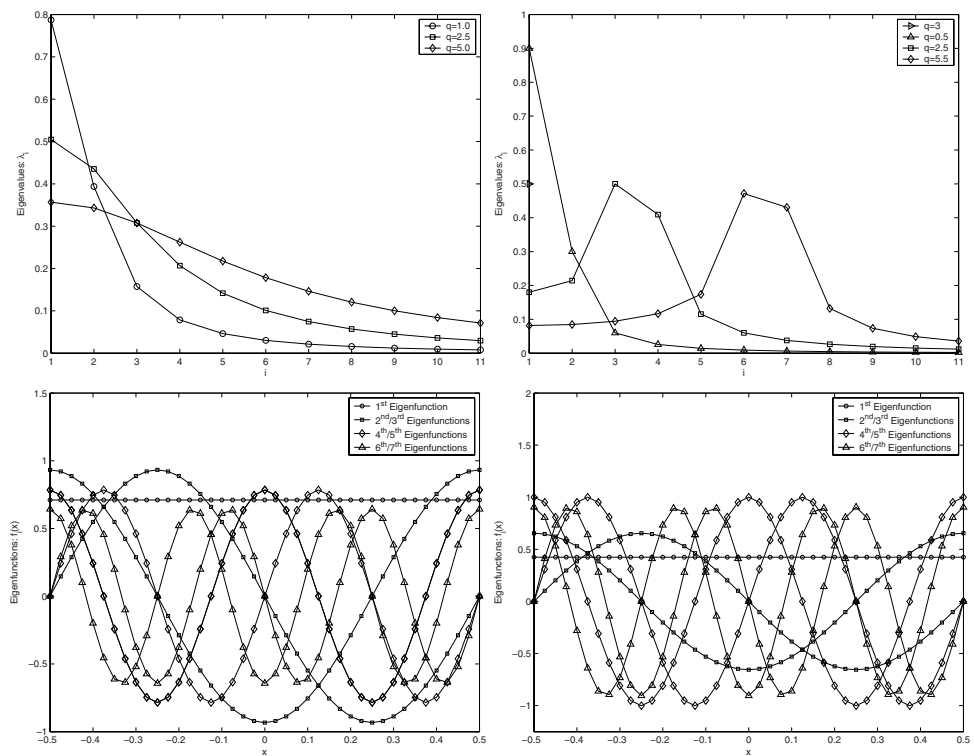

Fig. 1. Left column: Kernel1. Right column: Kernel2. Top row: Eigenvalues $\lambda_{i}$ for various values of the correlation length, here given by $q$, with $q=L /(2 \pi A)$ and $L=1$. Bottom row: First four-pairs eigenfunctions $f_{i}(x)$ with $|x| \leq L / 2$ and $L=1$ and $q=2.5$.
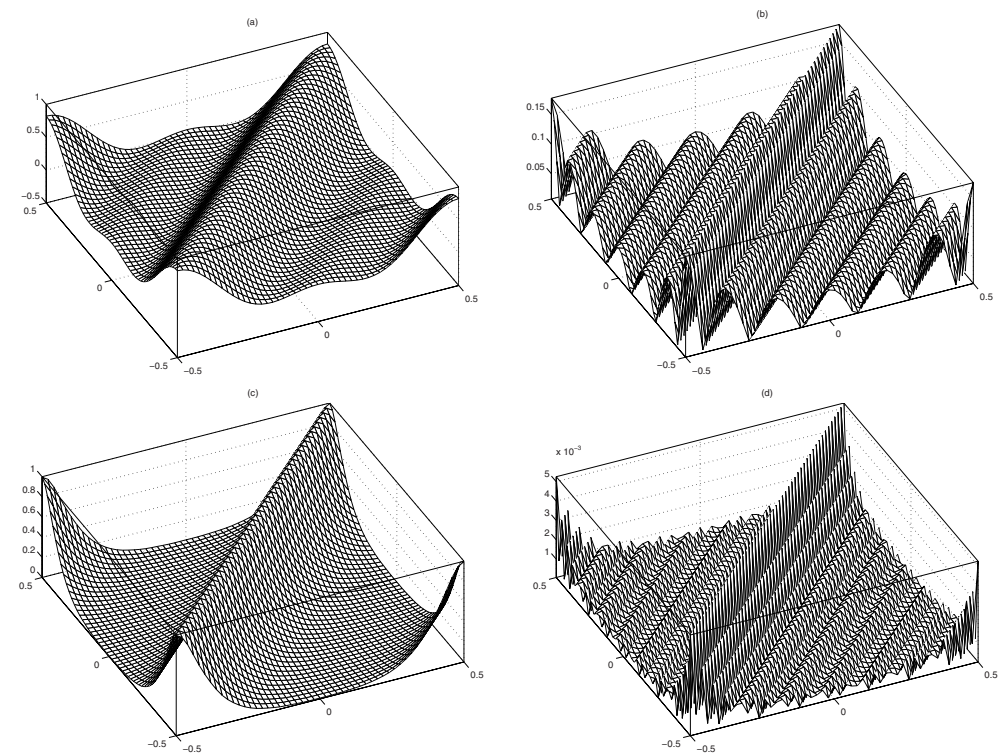

Fig. 2. Approximation of Covariance Kernel1 with $q=2.5 ; k=5 \pi$. (a): $n=5$-term $\mathrm{KL}$ approximation (b): $n=5$-term absolute error (c): $n=21$-term KL approximation (d): $n=21$-term absolute error. 

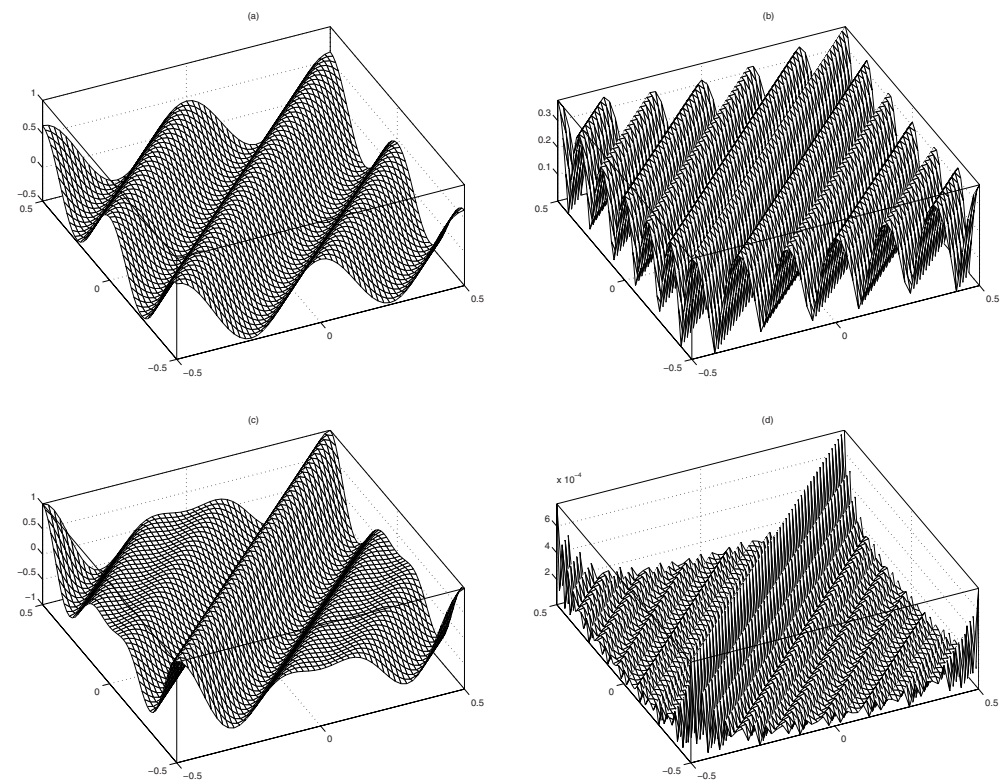

Fig. 3. Approximation of Covariance Kernel2 with $q=2.5 ; k=5 \pi,(\mathrm{a}): n=5$-term KL approximation (b): $n=5$-term absolute error (c): $n=21$-term KL approximation (d): $n=21$-term absolute error.

In the following, we will refer to the covariance kernel given by equation (20) as Kernel1. We show a close approximation to this kernel in Figure 2, This kernel is periodic, as expected, and the decay rate is controlled by the correlation length value. The kernel decays faster for smaller correlation lengths.

Similarly and under the same assumptions as in the previous case, if $b=$ $e^{\frac{1}{2}(k \Delta x)^{2}}>1$, which corresponds to equation (16) with a positive sign, it can be shown that the covariance kernel is similar to Kernel1 and becomes:

$$
\begin{aligned}
c(x, y)= & \frac{1}{2 \alpha+\sin (2 \alpha)}[2 \alpha+\sin (2 \alpha)+\alpha|x-y|(\cos (2 \alpha)-1)] \cos (\alpha|x-y|) \\
& +[1-\cos (2 \alpha)+\alpha|x-y| \sin (2 \alpha)] \sin (\alpha|x-y|) .
\end{aligned}
$$

In the following, we will refer to the covariance kernel given by equation (21) as Kernel2. We show a close approximation to this kernel in Figure 3 This kernel decays faster for smaller correlation lengths. Interestingly, if $q$ is an integer, the covariance kernel given by equation (21) simply becomes $c(x, y)=\cos (\alpha|x-y|)$. Since all those kernels are periodic, it is possible to employ the Fourier decomposition method of the integral equation described in the previous paragraph, in order to build a KL representation of the corresponding processes. In this case, the time dimension is treated as a spatial dimension without loss of generality. We obtain the eigenvalues and eigenfunctions of the kernels from equations (10) and (7,8). The integrals of equations (12) for Kernel1 and 
Kernel2 are computed numerically using numerical quadratures, except for the case of Kernel2 with $q$ being an integer. In that case, we can directly derive exact expressions for the eigenvalues and eigenfunctions of the kernel. We only have one double eigenvalue corresponding to two eigenfunctions:

$$
\lambda_{0}=\lambda_{1}=\frac{L}{2} ; \quad \psi^{(0)}=\sqrt{\frac{2}{L}} \cos (k x) \quad \text { and } \quad \psi^{(1)}=\sqrt{\frac{2}{L}} \sin (k x)
$$

Figure 1 fop shows the eigenvalues for Kernel1 (left) and for Kernel2 (right) for different correlation lengths $A$. Note that the smaller the value of $A$, the more contribution is from terms associated with smaller eigenvalues.

Figure 1bottom shows the first seven eigenfunctions for Kernel1 (left) and Kernel2 (right) for $q=2.5$. As seen in equations (10), each eigenvalue $\lambda_{n}$ is associated with a pair of eigenfunctions $\psi_{1}^{(n)}$ and $\psi_{2}^{(n)}$ corresponding to a cosine and sine contributions, respectively. Figures 2 and 3 show the 5-term and 21term KL approximations of Kernel1 and Kernel2 for $q=2.5$ and the associated errors. We notice that the kernels obtained from the KL representations tend to the corresponding exact kernels as the number of terms $n$ increases. For both cases with identical mode number $q$, the 21-term approximation does much better than the 5-term approximation. It is interesting to notice that the convergence is not monotonic, and the maximum absolute error is about one order of magnitude lower for Kernel2 than for Kernel1 for the 21-term approximation, see Figures 2 (d) and 3 (d).

Acknowledgements. This work was supported by the DDDAS program of the National Science Foundation under the supervision of Dr. Frederica Darema.

\section{References}

1. M. Loève. Probability Theory, Fourth edition. Springer-Verlag, 1977.

2. R.G. Ghanem and P. Spanos. Stochastic Finite Elements: a Spectral Approach. Springer-Verlag, 1991.

3. H. Stark and W.J. Woods. Probability, Random Process, and Estimation Theory for Engineers. Prentice-Hall, Inc., Englewood Cliffs, New Jersey, 1994.

4. P. Whittle. On stationary processes in the plane. Biometrika, 41:434-449, 1954.

5. S.P. Huang, S.T. Quek, and K.K. Phoon. Convergence study of the truncated Karhunen-Loeve expansion for simulation of stochastic processes. Int. J. Numer. Methods Eng., 52:1029-1043, 2001.

6. D. Lucor, C.-H. Su, and G.E. Karniadakis. Generalized polynomial chaos and random oscillators. Int. J. Numer. Methods Eng., in press, 2004.

7. M. Shinozuka and G. Deodatis. Simulation of the stochastic process by spectral representation. ASME Appl. Mech. Rev., 44(4):29-53, 1991.

8. M. Grigoriu. On the spectral representation method in simulation. Probabilistic Engineering Mechanics, 8(2):75-90, 1993.

9. D. Lucor. Generalized Polynomial Chaos: Applications to Random Oscillators and Flow-Structure Interactions. PhD thesis, Brown University, 2004.

10. C.H. Su and D. Lucor. Derivations of covariance kernels from dynamical systems and PDEs. In ICOSAHOM-04, Brown University, Providence USA, June 21-25, 2004 . 